\title{
Coadministration of a 9-Valent Human Papillomavirus Vaccine With Meningococcal and Tdap Vaccines
}

Andrea Schilling, MDa, Mercedes Macias Parra, MD ${ }^{b}$, Maricruz Gutierrez, MDc Jaime Restrepo, MD ${ }^{d}$, Santiago Ucros, MD ${ }^{e}$, Teobaldo Herrera, MDf, Eli Engel, MDs, Luis Huicho, MD ${ }^{h}$, Marcia Shew, MD', Roger Maansson, MS', Nicole Caldwell, BS ${ }^{j}$, Alain Luxembourg, MD, PhD', Ajoke Sobanjo-ter Meulen, MDj

BACKGROUND: This study in 11- to 15-year-old boys and girls compared the immunogenicity and safety of GARDASIL 9 (9-valent human papillomavirus [9vHPV] vaccine) administered either concomitantly or nonconcomitantly with 2 vaccines routinely administered in this age group (Menactra [MCV4; Neisseria meningitidis serotypes A/C/Y/W-135] or Adacel [Tdap; diphtheria/tetanus/acellular pertussis]).

метнорS: Participants received 9vHPV vaccine at day 1 and months 2 and 6; the concomitant group $(n=621)$ received MCV4/Tdap concomitantly with 9vHPV vaccine at day 1 ; the nonconcomitant group $(n=620)$ received MCV4/Tdap at month 1. Antibodies to HPV-, MCV4-, and Tdap-relevant antigens were determined. Injection-site and systemic adverse events (AEs) were monitored for 15 days after any vaccination; serious AEs were monitored throughout the study.

RESULTS: The geometric mean titers for all HPV types in 9vHPV vaccine 4 weeks after dose 3, proportion of subjects with a fourfold rise or greater in titers for $4 \mathrm{~N}$ meningitidis serotypes 4 weeks after injection with MCV4, proportion of subjects with antibody titers to diphtheria and tetanus $\geq 0.1 \mathrm{IU} / \mathrm{mL}$, and geometric mean titers for pertussis antigens 4 weeks after injection with Tdap were all noninferior in the concomitant group compared with the nonconcomitant group. Injection-site swelling occurred more frequently in the concomitant group. There were no vaccine-related serious AEs.

concLusions: Concomitant administration of 9vHPV vaccine with MCV4/Tdap was generally well tolerated and did not interfere with the antibody response to any of these vaccines. This strategy would minimize the number of visits required to deliver each vaccine individually.

\footnotetext{
${ }^{a}$ Facultad de Medicina Clinica Alemana-Universidad del Desarrollo, Santiago, Chile; ${ }^{b}$ Instituto Nacional de Pediatría, Mexico City, Mexico; ${ }^{C}$ Hospital del Niño Poblano, Puebla, Mexico; ${ }^{d}$ Fundacion Centro de Investigacion Clinica CIC, Medellín, Colombia; ${ }^{e}$ Centro de Investigaciones en Salud, Fundacion Santa Fe de Bogotá, Bogotá, Colombia; Insituto de Investigación Nutricional anexo Huáscar, Lima, Perú; ${ }^{g}$ Bayview Research Group, Valley Village, California; ' Instituto Nacional de Salud del Niño, Lima, Perú; 'Indiana University School of Medicinel Department of Pediatrics, Indianapolis, Indiana; and ${ }^{j}$ Merck \& Co., Inc., Kenilworth, New Jersey
}

Dr Schilling was the lead clinical author; substantially contributed to acquisition of the data, interpretation of the results, and drafting of the manuscript; and reviewed and revised the manuscript for important intellectual content; Drs Parra, Gutierrez, Restrepo, Ucros, and Engel substantially contributed to acquisition of the data and reviewed and revised the manuscript for important intellectual content; Dr Herrera substantially contributed to acquisition of the data and drafting of the manuscript and reviewed and revised the manuscript for important intellectual content; Dr Shew substantially contributed to acquisition of the data and interpretation of the results and reviewed and revised the manuscript for important intellectual content; Mr Maansson substantially contributed to analysis of the data and reviewed and revised the manuscript for important intellectual content; Ms Caldwell substantially contributed to conception, design, and planning of the study and acquisition of the data and reviewed and revised the manuscript for important intellectual content;
WHAT'S KNOWN ON THIS SUBJECT: Previous studies have shown that concomitant administration of the quadrivalent human papillomavirus vaccine with MCV4 and Tdap was generally well tolerated and did not interfere with the immune responses to the respective vaccines.

WHAT THIS STUDY ADDS: Concomitant administration of the novel 9-valent human papillomavirus vaccine with MCV4 and Tdap, 2 vaccines that are currently recommended for routine vaccination of adolescents, did not compromise the safety, tolerability, and immunogenicity of the individual vaccines. 
A 9-valent (6/11/16/18/31/33/45/ 52/58) human papillomavirus (HPV; $9 \mathrm{vHPV}$ ) vaccine was developed to cover 7 cancer-causing HPV types (HPV 16, 18, 31, 33, 45, 52, 58) that are together responsible for $\sim 90 \%$ of cervical cancers and HPV-related vulvar, vaginal, and anal cancers, and 2 HPV types (HPV 6 and 11) that are responsible for $90 \%$ of genital warts. ${ }^{1-5}$ In clinical studies, the $9 \mathrm{vHPV}$ vaccine prevented persistent infection and disease due to the HPV vaccine types in females 16 to 26 years of age ${ }^{6}$; efficacy findings were extended to girls and boys 9 to 15 years of age based on noninferior immunogenicity. ${ }^{7}$ The $9 v \mathrm{vHPV}$ vaccine was licensed in the United States in December 2014 under the name GARDASIL9 (Merck \& Co., Inc., Kenilworth, NJ)..$^{8}$ In February 2015, the Advisory Committee on Immunization Practices included GARDASIL9 in its recommendations for routine HPV vaccination of boys and girls at age 11 or 12 years and catchup vaccination in females 13 to 26 years and males 13 to 21 years not vaccinated previously. ${ }^{9}$ Epidemiologic studies have demonstrated the acquisition of HPV soon after sexual initiation. ${ }^{10}$ The median age of sexual debut is in the late teens (15-19 years) in most countries. ${ }^{11}$ Thus, preadolescent boys and girls $\leq 15$ years of age represent the ideal HPV vaccination population.

Most vaccine schedules worldwide rely on the concomitant administration of vaccines to improve the adherence to vaccination and to lower the cost of the programs, both in childhood and in adolescent vaccination. Because adolescents and adults need to maintain the protection given by several childhood vaccines, such as diphtheria, tetanus, and whooping cough, and need to be protected against Neisseria meningitidis before exposure to new populations such as those in high schools, college, or military service, etc, a booster dose of the diphtheria, tetanus, and acellular pertussis vaccines and routine vaccination for meningococcal serogroups $\mathrm{A} / \mathrm{C} / \mathrm{Y} /$ $\mathrm{W}-135$ are recommended for adolescents in several countries. ${ }^{12-14}$ A logical step was to include the $9 \mathrm{vHPV}$ vaccine in those alreadyestablished programs.

This study in boys and girls 11 to 15 years of age was designed to evaluate the immunogenicity and safety of the concomitant administration of a first dose of the 9vHPV vaccine with MCV4 (Menactra [meningococcal polysaccharide conjugate serogroups A/C/Y/W-135, Sanofi-Pasteur Inc, Lyon France]) and Tdap (Adacel [tetanus, diphtheria, acellular pertussis, SanofiPasteur Inc]), 2 vaccines that are also recommended by the Advisory Committee on Immunization Practices for routine vaccination of preadolescents and adolescents and thereby help inform decisions by those who would be administering these vaccines together (Merck Protocol V503-005; NCT00988884).

\section{METHODS}

\section{Study Population}

Between October 22, 2009, and February 22, 2011, 1241 healthy boys and girls aged 11 to 15 who denied any sexual activity (and who were not planning on becoming sexually active through the course of the study) from 41 sites located in Chile $(n=100)$, Colombia $(n=140)$, Mexico $(n=200)$, Peru $(n=100)$, and the United States $(n=701)$ participated in the study. Reasons for exclusion from the study included pregnancy (determined by urine or serum $\beta$-human chorionic gonadotropin testing), known allergy to any vaccine component, thrombocytopenia, and immunosuppression/prior immunosuppressive therapy or previous receipt of an HPV vaccine. Subjects must not have been immunized against diphtheria, tetanus, and pertussis in the past 5 years or received a meningococcal vaccine. The study was conducted in conformity with applicable national or local requirements regarding ethical committee review, informed consent, and the protection of the rights and welfare of human subjects participating in biomedical research. An external data monitoring committee assessed safety findings throughout the study.

\section{Study Design}

This was an open-label, randomized, multicenter, comparative study.

Subjects were stratified by gender $(1: 1$ ratio) and randomly assigned to 1 of 2 vaccination groups (concomitant group [Group A] or nonconcomitant group [Group B]) in a 1:1 ratio. At day 1, subjects in Group A received the first dose of 9vHPV vaccine in the deltoid muscle of the nondominant arm and MCV4 and Tdap in the deltoid muscle of the opposite arm. Subjects in Group B received the first dose of the 9vHPV vaccine on day 1 in the nondominant arm and MCV4 and Tdap 1 month later (month 1) in the dominant arm. All subjects received the second dose of the 9vHPV vaccine at month 2 and the third dose at month 6 . The compositions of the 3 vaccines have been described previously.6,15,16

Blood samples were drawn immediately before vaccination at day 1, month 1, month 2 (Group B only), and month 7. Serum collected from all subjects at day 1 and month 7 underwent analysis of anti-HPV responses with a competitive Luminex Immunoassay, performed by PPD Vaccines and Biologics (Wayne, PA). ${ }^{17}$ Serum collected at day 1 and month 1 for Group A and month 1 and month 2 for Group B underwent antibody testing for $N$ meningitidis serogroups A/C/Y/W-135, diphtheria, tetanus, and pertussis. $N$ meningitidis serogroups $\mathrm{A} / \mathrm{C} / \mathrm{Y} / \mathrm{W}-135$ serum bactericidal antibody assay was performed by the Health Protection Agency, Manchester Medical Microbiology Partnership (Manchester, United Kingdom). Diphtheria antibodies were measured by a diphtheria antitoxin cell culture assay performed by CSL Limited 
TABLE 1 Noninferiority Criteria Corresponding to the Primary Hypotheses Related to Immunogenicity

\begin{tabular}{|c|c|c|c|c|}
\hline Antigen & Paramater $^{\mathrm{a}}$ & Expected Rates/SDs & Noninferiority Margin & Power \\
\hline HPV type $6,11,16,18,31,33,45,52,58^{\text {b }}$ & GMT & $\sigma=1.2$ & Twofold decrease & $>99.9 \%$ \\
\hline N meningitidis Serogroup A & $\%$ with fourfold or greater rise in titer & $90 \%$ & 10 percentage points & $99.8 \%$ \\
\hline N meningitidis Serogroup C & $\%$ with fourfold or greater rise in titer & $90 \%$ & 10 percentage points & $99.8 \%$ \\
\hline$N$ meningitidis Serogroup $Y$ & $\%$ with fourfold or greater rise in titer & $80 \%$ & 10 percentage points & $95.1 \%$ \\
\hline N meningitidis Serogroup W-135 & $\%$ with fourfold or greater rise in titer & $95 \%$ & 10 percentage points & $>99.9 \%$ \\
\hline Diphtheria & $\%$ with titer $>0.1 \mathrm{IU} / \mathrm{mL}$ & $95 \%$ & 10 percentage points & $>99.9 \%$ \\
\hline Tetanus & $\%$ with titer $>0.1 \mathrm{IU} / \mathrm{mL}$ & $92 \%$ & 10 percentage points & $99.8 \%$ \\
\hline Pertussis PT & GMT & $\sigma=1.0$ & 1.5 -fold decrease & $>99.9 \%$ \\
\hline Pertussis FHA & GMT & $\sigma=0.8$ & 1.5 -fold decrease & $>99.9 \%$ \\
\hline Pertussis PRN & GMT & $\sigma=1.3$ & 1.5 -fold decrease & $>99.9 \%$ \\
\hline Pertussis FIM & GMT & $\sigma=1.5$ & 1.5 -fold decrease & $97.0 \%$ \\
\hline
\end{tabular}

a Noninferiority of anti-HPV GMTs was measured at 4 weeks after dose 3 of 9vHPV vaccine. Noninferiority of $N$ meningitidis serogroups A/C/Y/W-135, diphtheria, tetanus, and pertussis was measured 4 weeks postvaccination with MCV4 and Tdap.

b Each HPV type is tested separately

(Parkville, Australia) and calibrated against the First International Standard for Diphtheria Antitoxin. Tetanus antibodies were measured by a tetanus antitoxin enzyme immunoassay performed by CSL Limited and calibrated against the First International Standard for Tetanus Immunoglobulin. Pertussis antibodies were measured by an anti-pertussis toxin (PT) enzyme-linked immunosorbent assay (ELISA), anti-filamentous hemagglutinin (FHA) ELISA, anti-pertactin (PRN) ELISA, and anti-fimbriae 2/3 (FIM) ELISA performed by Michael E. Pichichero Laboratory, Rochester General Hospital Research Institute (Rochester, NY). The anti-PT ELISA, anti-FHA ELISA, and anti-FIM ELISA were calibrated against the US Food and Drug Administration Pertussis reference lot 3 , and the anti-PRN ELISA was calibrated against the US Food and Drug Administration Pertussis reference lot 4 .

\section{Safety Measurements}

All subjects received a vaccination report card (VRC) at the day 1 and months 1, 2, and 6 visits. VRCs for all the subjects were to be completed after the month-1 visit to provide a common period of follow-up even though subjects in the Group A were not vaccinated at month 1 . On the VRC, the parent/guardian was asked to record (1) the subject's oral temperature in the evening of the day of each study vaccination and daily for a total of 5 days and (2) injection-site and systemic adverse events (AEs) for a total of 15 days including the day of vaccination after each study vaccination. Serious AEs were collected for the whole duration of the study regardless of causality and were followed for outcome. For all injection-site AEs, except erythema and swelling, subjects were instructed by the VRC to estimate the severity of AEs as mild (awareness of symptom but easily tolerated), moderate (discomfort enough to cause interference with usual activities), or severe (incapacitating with inability to work or do usual activity). For erythema and swelling, subjects were instructed by the VRC to measure an injection-site reaction at its greatest width ("maximum size") from edge to edge in maximum units ranging from 0 to $>7$ inches $(17.5 \mathrm{~cm})$ on the VRC, rounding up to the next unit if in between 2 units (each unit on the VRC measured $\sim 1$ inch $[2.5 \mathrm{~cm}])$.

\section{Statistical Analysis}

Primary immunogenicity analyses were done "per-protocol". Subjects in perprotocol analyses for the $9 \mathrm{vHPV}$ vaccine analyses had to receive all 3 doses of $9 \mathrm{vHPV}$ vaccine within acceptable day ranges and 1 dose of MCV4 and Tdap. In addition, for per-protocol analysis for $9 \mathrm{vHPV}$ vaccine, subjects had to (1) have at least 1 postdose 3 serology result within acceptable day ranges; (2) in analyses for the HPV6 and HPV11 components, be seronegative to both

TABLE 2 Summary of Subject Characteristics by Vaccination Group at Enrollment

\begin{tabular}{|c|c|c|}
\hline Characteristic & $\begin{array}{c}\text { Group } A^{\text {a }} \text { (Concomitant) } \\
(N=621)\end{array}$ & $\begin{array}{c}\text { Group } \mathrm{B}^{\mathrm{a}} \text { (Nonconcomitant) } \\
(N=620)\end{array}$ \\
\hline & $n(\%)$ & $n(\%)$ \\
\hline \multicolumn{3}{|l|}{ Gender } \\
\hline Male & $310(49.9)$ & $310(50.0)$ \\
\hline Female & $311(50.1)$ & $310(50.0)$ \\
\hline \multicolumn{3}{|l|}{ Age (y) } \\
\hline Mean (SD) & $12.2(1.4)$ & $12.1(1.3)$ \\
\hline Median (range) & $12.0(11$ to 15$)$ & $12.0(11$ to 15$)$ \\
\hline \multicolumn{3}{|l|}{ Region } \\
\hline North America & $344(55.4)$ & $357(57.6)$ \\
\hline Latin America & $277(44.6)$ & $263(42.4)$ \\
\hline \multicolumn{3}{|l|}{ Race } \\
\hline American Indian or Alaska Native & $60(9.7)$ & $59(9.5)$ \\
\hline Asian & $6(1.0)$ & $8(1.3)$ \\
\hline Black or African American & $38(6.1)$ & $41(6.6)$ \\
\hline Multiracial & $217(34.9)$ & $217(35.0)$ \\
\hline Native Hawaiian or 0ther Pacific Islander & $2(0.3)$ & $5(0.8)$ \\
\hline White & $298(48.0)$ & $290(46.8)$ \\
\hline
\end{tabular}

a Group A (concomitant administration) received a 0.5-mL dose of 9vHPV vaccine at day 1, month 2, and month 6 and MCV4 and Tdap on day 1; Group B (nonconcomitant administration) received 9vHPV vaccine as above and MCV4 and Tdap at month 1. 
HPV6 and 11 at day 1; (3) in analyses for the other vaccine HPV types, be seronegative at day 1 only for the HPV type being analyzed; and (4) have no protocol violations that were considered to affect the immune responses. For per-protocol analysis for MCV4 and Tdap, subjects had to (1) have received MCV4 and Tdap within acceptable day ranges; (2) have at least 1 serology result after administration of MCV4 and Tdap within acceptable day ranges; and (3) have no protocol violations that were considered to affect the immune responses.

The primary and secondary end points for evaluating antibody responses to the 9vHPV vaccine were geometric mean titers (GMTs) to HPV6/11/16/18/31/ $33 / 45 / 52 / 58$ and the percentages of subjects who seroconverted for each HPV type by 4 weeks after the third dose of 9vHPV vaccine. Anti-HPV cutoffs for determining serostatus were $30,16,20,24,10,8,8,8$, and 8 milliMerck units/mL for HPV types 6, 11, $16,18,31,33,45,52$, and 58, respectively. The primary end points for evaluating antibody response to MCV4 and Tdap were the proportions of subjects who achieved acceptable serological responses to $N$ meningitidis serogroups A/C/Y/W-135, diphtheria, and tetanus and GMTs for pertussis (anti-PT, anti-FHA, anti-PRN, and anti-FIM) 4 weeks postvaccination with MCV4 and Tdap.

Noninferiority criteria for each immunogenicity hypothesis are shown in Table 1. Noninferiority of anti-HPV GMTs 4 weeks postdose 3 and pertussis GMTs 4 weeks postvaccination with MCV4 and Tdap was based on 1-sided tests of noninferiority (conducted at the 0.025 significance level) comparing GMTs between Group A and Group B for each component. An analysis of variance model (1 for each component) was used with a response of $\log _{\mathrm{e}}$ individual titers and fixed effects for vaccination group and gender. Noninferiority of anti-HPV seroconversion rates and of serologic responses to $N$ meningitidis serogroups
TABLE 3 Summary of Exclusions ${ }^{\mathrm{a}}$ From the Per-Protocol Immunogenicity Populations for 9vHPV Vaccine and MCV4 and Tdap

\begin{tabular}{|c|c|c|}
\hline & $\begin{array}{c}\text { Concomitant } \\
\text { (Group A), } \\
N=619\end{array}$ & $\begin{array}{c}\text { Nonconcomitant } \\
\text { (Group B), } \\
N=618\end{array}$ \\
\hline \multicolumn{3}{|l|}{ Exclusions for the 9vHPV vaccine } \\
\hline Number randomized who received $\geq 1$ injection, $n$ & 619 & 618 \\
\hline \multicolumn{3}{|l|}{$\begin{array}{l}\text { Number excluded from the per-protocol immunogenicity } \\
\text { analyses, } n(\%)\end{array}$} \\
\hline HPV6/11 & 117 (18.9) & $104(16.8)$ \\
\hline HPV16 & $106(17.1)$ & $88(14.2)$ \\
\hline HPV18 & $103(16.6)$ & $83(13.4)$ \\
\hline HPV31 & $105(17.0)$ & $82(13.3)$ \\
\hline HPV33 & $99(16.0)$ & $81(13.1)$ \\
\hline HPV45 & $96(15.5)$ & $79(12.8)$ \\
\hline HPV52 & $98(15.8)$ & $80(12.9)$ \\
\hline HPV58 & $100(16.2)$ & $81(13.1)$ \\
\hline \multicolumn{3}{|l|}{ Reasons for exclusion, $n$ (\%) } \\
\hline Did not receive MCV4 or Tdap & $0(0.0)$ & $18(2.9)$ \\
\hline Received incorrect clinical material & $0(0.0)$ & $2(0.3)$ \\
\hline Received nonstudy vaccination ${ }^{\mathrm{C}}$ & $6(1.0)$ & $8(1.3)$ \\
\hline Received immunosuppressives before month 7 & $0(0.0)$ & $2(0.3)$ \\
\hline Incorrectly randomized & $2(0.3)$ & $0(0.0)$ \\
\hline History of immune disorder & $1(0.2)$ & $3(0.5)$ \\
\hline Did not complete the 3-dose regimen of 9vHPV vaccine & $32(5.2)$ & $29(4.7)$ \\
\hline $\begin{array}{l}\text { Received } \geq 1 \text { dose of } 9 \mathrm{vHPV} \text { vaccine outside the } \\
\text { acceptable day range }\end{array}$ & $10(1.6)$ & $7(1.1)$ \\
\hline Serum sample or results missing at day 1 & $3(0.5)$ & $2(0.3)$ \\
\hline Serum sample or results missing at month 7 & $78(12.6)$ & $60(9.7)$ \\
\hline Day 1 seropositive, ${ }^{\mathrm{d}} n(\%)$ & & n (\%) \\
\hline $\mathrm{HPV} 6 / 11^{\mathrm{e}}$ & $27(4.4)$ & $31(5.0)$ \\
\hline HPV16 & $13(2.1)$ & $16(2.6)$ \\
\hline HPV18 & $10(1.6)$ & $8(1.3)$ \\
\hline HPV31 & $11(1.8)$ & $5(0.8)$ \\
\hline HPV33 & $5(0.8)$ & $4(0.6)$ \\
\hline HPV45 & $2(0.3)$ & $2(0.3)$ \\
\hline HPV52 & $4(0.6)$ & $3(0.5)$ \\
\hline HPV58 & $9(1.5)$ & $5(0.8)$ \\
\hline \multicolumn{3}{|l|}{ Exclusions for MCV4 and Tdap } \\
\hline Number randomized who received $\geq 1$ injection & 619 & 618 \\
\hline $\begin{array}{l}\text { Number excluded from the per-protocol immunogenicity } \\
\text { analyses, } n(\%)\end{array}$ & $24(3.9)$ & $52(8.4)$ \\
\hline \multicolumn{3}{|l|}{ Reason for exclusion, $n(\%)$} \\
\hline Did not receive MCV4 or Tdap & $0(0.0)$ & $18(2.9)$ \\
\hline Received incorrect clinical material & $0(0.0)$ & $2(0.3)$ \\
\hline Received nonstudy vaccination ${ }^{\mathrm{C}}$ & $3(0.5)$ & $8(1.3)$ \\
\hline Received immunosuppressives before month 7 & $0(0.0)$ & $2(0.3)$ \\
\hline Incorrectly randomized & $2(0.3)$ & $0(0.0)$ \\
\hline With history of immune disorder & $1(0.2)$ & $3(0.5)$ \\
\hline $\begin{array}{l}\text { Received MCV4 and Tdap outside the acceptable day } \\
\text { range }\end{array}$ & $0(0.0)$ & $13(2.1)$ \\
\hline $\begin{array}{l}\text { Serum sample or results missing at } 4 \text { wk postinjection } \\
\text { of Tdap-IPV vaccine }\end{array}$ & $18(2.9)$ & $31(5.0)$ \\
\hline Received incorrect Tdap dose ${ }^{f}$ & $0(0.0)$ & $1(0.2)$ \\
\hline
\end{tabular}

a subject may appear in $>1$ category.

b Group A (concomitant administration) received a $0.5-\mathrm{mL}$ dose of 9vHPV vaccine at day 1, month 2, and month 6 , and MCV4 and Tdap on day 1; Group B (nonconcomitant administration) received 9vHPV vaccine as with Group A together with MCV4 and Tdap at month 1.

${ }^{c}$ Includes (1) any live vaccine received $21 \mathrm{~d}$ before or $14 \mathrm{~d}$ after study vaccine or (2) any inactivated or recombinant vaccine received within $14 \mathrm{~d}$ of study vaccine.

d Seropositive at day 1 to the relevant HPV types(s) applies to the per protocol population for the relevant HPV type(s) only e Because the L1 proteins for HPV6 and HPV11 are 92\% homologous at the amino acid level, all participants were required to be seronegative to both HPV6 and HVP11 to be included in either of the HPV6 and HPV11 per-protocol immunogenicity populations.

f Excluded from the per protocol immunogenicity population for Tdap; not excluded from the per protocol immunogenicity population for MCV4. 
A/C/Y/W-135, diphtheria, and tetanus was tested by 1-sided tests of noninferiority comparing proportions between Group A and Group B for each component. These tests were conducted based on methods developed by Miettinen and Nurminen. ${ }^{18}$ All tests were conducted at the 0.025 significance level. Success in this study was declared if the primary hypotheses of noninferiority were demonstrated for all components of 9vHPV vaccine and at least 1 of MCV4 and Tdap. With 620 subjects per group (1240 total), this study had an overall power $>99 \%$ for the primary immunogenicity hypotheses (Table 1).

All subjects who received at least 1 study vaccination and had follow-up data were included in safety analyses. AEs were summarized descriptively as frequencies and percentages by participant groups.

\section{RESULTS}

A total of 1254 subjects residing in Latin America and North America were screened for inclusion in this study and 1241 were randomized (621 to Group A and 620 to Group B). The numbers of subjects who were randomized, vaccinated, and completed or discontinued the study are shown in Supplemental Figure 1. Both vaccination groups were comparable with respect to baseline demographics (Table 2). Approximately 6.4\% (79 of 1241) of patients were African American, and $1.1 \%$ (14/1241) were Asian.

The most common reason for exclusion from the per-protocol analyses was having a serum sample or result missing at 4 weeks postinjection (Table 3). Few subjects $(0.3 \%-4.7 \%)$ were excluded from the per-protocol analyses for 9vHPV vaccine because of testing positive for HPV on Day 1.

Month 7 anti-HPV GMTs against all HPV types were comparable in Group A and Group B, with fold differences (ie, Group A/Group B) ranging from 0.97 for HPV6 and HPV11 to 1.10 for HPV45 (Table 4). The noninferiority

TABLE 4 Anti-HPV GMTs and Estimated Fold Difference at 4 Weeks After Dose 3 in the HPV PerProtocol Populations

\begin{tabular}{|c|c|c|c|c|c|}
\hline \multirow[t]{2}{*}{ Antigen } & \multicolumn{2}{|r|}{$\begin{array}{c}\text { Concomitant (Group A), } \\
\qquad N=619\end{array}$} & \multicolumn{2}{|c|}{$\begin{array}{l}\text { Nonconcomitant (Group B), }{ }^{\mathrm{a}} \\
\qquad N=618\end{array}$} & \multirow{2}{*}{$\begin{array}{l}\text { Estimated Fold } \\
\text { Difference Group A/Group B } \\
\text { (95\% Confidence Interval) }\end{array}$} \\
\hline & $n$ & Estimated GMT $(\mathrm{mMU} / \mathrm{mL})$ & $n$ & Estimated GMT $(\mathrm{mMU} / \mathrm{mL})$ & \\
\hline HPV6 & 501 & 2198.7 & 514 & 2260.7 & 0.97 (0.88 to 1.08$)$ \\
\hline HPV11 & 502 & 1495.0 & 514 & 1547.2 & $0.97(0.87$ to 1.07$)$ \\
\hline HPV16 & 513 & 8882.6 & 530 & 9027.6 & 0.98 (0.89 to 1.09$)$ \\
\hline HPV18 & 516 & 2610.4 & 535 & 2633.9 & 0.99 (0.88 to 1.12$)$ \\
\hline HPV31 & 514 & 2439.4 & 536 & 2334.3 & $1.04(0.93$ to 1.17$)$ \\
\hline HPV33 & 520 & 1268.5 & 537 & 1276.3 & 0.99 (0.89 to 1.11$)$ \\
\hline HPV45 & 523 & 947.8 & 539 & 863.8 & $1.10(0.97$ to 1.25$)$ \\
\hline HPV52 & 521 & 1082.7 & 538 & 1103.7 & $0.98(0.88$ to 1.10$)$ \\
\hline HPV58 & 519 & 1532.8 & 537 & 1555.1 & $0.99(0.88$ to 1.10$)$ \\
\hline
\end{tabular}

mMU, milliMerck units; $N$, number of subjects randomized to the respective vaccination group who received $\geq 1$ injection $n=$ number of subjects contributing to the analysis.

a Group A (concomitant administration) received a 0.5-mL dose of 9vHPV vaccine at day 1, month 2, and month 6 and MCV4 and Tdap on day 1; Group B (nonconcomitant administration) received 9vHPV vaccine as Group A and MCV4 and Tdap at month 1.

b $P$ value for noninferiority $<.001$ for all 9 antigens. The noninferiority criterion for GMT end points reported in this table was defined as statistically less than a twofold decrease in Group A compared with Group B. Noninferiority of GMT in Group A relative to Group B was demonstrated if the lower limit of the $95 \%$ confidence interval for the fold difference was $>0.5$.

criteria for the anti-HPV GMT responses in Group A relative to Group B were achieved. Seroconversion rates were $100 \%$ for all HPV types in both groups and were noninferior in Group A compared with Group B (Table 5).

At least $75 \%$ of subjects achieved a fourfold or higher rise in titers to $N$ meningitidis serogroup A in both Group A and Group B, and at least 89\% of subjects achieved a fourfold or higher rise in titers to $N$ meningitidis

TABLE 5 Seroconversion Rates and Estimated Percent Difference at 4 Weeks After Dose 3 in the HPV Per-Protocol Populations

\begin{tabular}{|c|c|c|c|c|c|}
\hline \multirow[t]{2}{*}{ Antigen } & \multicolumn{2}{|c|}{$\begin{array}{c}\text { Concomitant } \\
\left(\text { Group A), }{ }^{a} N=619\right.\end{array}$} & \multicolumn{2}{|c|}{$\begin{array}{c}\text { Nonconcomitant } \\
\text { (Group B), }{ }^{a} N=618\end{array}$} & \multirow{2}{*}{$\begin{array}{c}\text { Estimated Percent Difference } \\
\text { Group A - Group B } \\
\text { (95\% confidence interval) }\end{array}$} \\
\hline & $m / n$ & Response (\%) & $m / n$ & Response (\%) & \\
\hline HPV6 & $501 / 501$ & 100 & $514 / 514$ & 100 & 0.0 (-0.8 to 0.7$)$ \\
\hline HPV11 & $502 / 502$ & 100 & $514 / 514$ & 100 & 0.0 (-0.8 to 0.7$)$ \\
\hline HPV16 & $513 / 513$ & 100 & $530 / 530$ & 100 & $0.0(-0.7$ to 0.7$)$ \\
\hline HPV18 & $516 / 516$ & 100 & $535 / 535$ & 100 & $0.0(-0.7$ to 0.7$)$ \\
\hline HPV31 & $514 / 514$ & 100 & $536 / 536$ & 100 & $0.0(-0.7$ to 0.7$)$ \\
\hline HPV33 & $520 / 520$ & 100 & $537 / 537$ & 100 & $0.0(-0.7$ to 0.7$)$ \\
\hline HPV45 & $523 / 523$ & 100 & $539 / 539$ & 100 & $0.0(-0.7$ to 0.7$)$ \\
\hline HPV52 & $521 / 521$ & 100 & $538 / 538$ & 100 & $0.0(-0.7$ to 0.7$)$ \\
\hline HPV58 & $519 / 519$ & 100 & $537 / 537$ & 100 & $0.0(-0.7$ to 0.7$)$ \\
\hline
\end{tabular}

$m=$ number of subjects with the indicated response; $N$, number of subjects randomized to the respective vaccination group who received at least 1 injection; $n$, number of subjects contributing to the analysis. Seropositive is defined as antiHPV serum levels (assessed by competitive Luminex immunoassay) greater or equal to $30,16,20,24,10,8,8,8,8$ milliMerck units/mL for HPV types $6,11,16,18,31,33,45,52$, and 58 , respectively.

a Group A (concomitant administration) received a 0.5-mL dose of 9vHPV vaccine at day 1, month 2, and month 6 and MCV4 and Tdap on day 1; Group B (nonconcomitant administration) received 9vHPV vaccine as Group A and MCV4 and Tdap at month 1.

b $P$ value for noninferiority $<.001$ for all 9 antigens. The noninferiority criterion for seroconversion end points reported in this table was defined as statistically $<5$ percentage points decrease in Group A compared with Group B. Noninferiority of seroconversion rates in Group A relative to Group B was demonstrated if the lower limit of the $95 \%$ confidence interval for the percentage point difference was greater than -5 . 
TABLE 6 Estimated Percentage Point Difference in the Per-Protocol Population for MCV4 for Percent of Subjects With Fourfold or Greater Rise in Titers for $N$ meningitidis Serogroups at 4 Weeks Postvaccination

\begin{tabular}{|c|c|c|c|c|c|c|c|}
\hline & \multicolumn{3}{|c|}{ Concomitant (Group A), ${ }^{a} N=619$} & \multicolumn{3}{|c|}{ Nonconcomitant (Group B), ${ }^{\mathrm{a}} N=618$} & \multirow{2}{*}{$\begin{array}{l}\text { Estimated Percentage Point Difference } \\
\text { Group A - Group B }(97.5 \% \mathrm{Cl})\end{array}$} \\
\hline & $m / n$ & $\%$ & $97.5 \% \mathrm{Cl}$ & $m / n$ & $\%$ & $97.5 \% \mathrm{Cl}$ & \\
\hline Serogroup A & $466 / 590$ & 79.0 & (75.0 to 82.6 ) & $425 / 564$ & 75.4 & (71.0 to 79.3 ) & $3.8(-1.7 \text { to } 9.3)^{\mathrm{b}}$ \\
\hline Serogroup C & $548 / 590$ & 92.9 & (90.1 to 95.1$)$ & $538 / 566$ & 95.1 & (92.6 to 96.9$)$ & $-2.1(-5.4 \text { to } 1.1)^{\mathrm{b}}$ \\
\hline Serogroup W-135 & $563 / 589$ & 95.6 & (93.3 to 97.3 ) & $553 / 566$ & 97.7 & (95.9 to 98.9$)$ & $-2.1(-4.7 \text { to } 0.3)^{\mathrm{b}}$ \\
\hline Serogroup Y & $540 / 590$ & 91.5 & (88.6 to 93.9 ) & $506 / 566$ & 89.4 & (86.1 to 92.1 ) & $2.1(-1.8 \text { to } 6.1)^{b}$ \\
\hline
\end{tabular}

$\mathrm{m}=$ number of subjects with the indicated response; $\mathrm{N}$, number of subjects randomized to the respective vaccination group who received at least 1 injection; $\mathrm{n}$, number of subjects contributing to the analysis. $\mathrm{Cl}$, confidence interval.

a Group A (concomitant administration) received a 0.5-mL dose of 9vHPV vaccine at day 1, month 2, and month 6 and MCV4 and Tdap on day 1; Group B (nonconcomitant administration) received 9vHPV vaccine as above and Tdap-IPV vaccine at month 1.

b $P$ value for noninferiority $<.001$ for all antigens. The noninferiority criterion for end points reported in this table was defined as statistically $<10$ percentage points decrease in Group A compared with Group B. Noninferiority of percent of subjects with fourfold or greater rise in titers for $N$ meningitidis serogroups in Group A relative to Group B was demonstrated if the lower limit of the $97.5 \% \mathrm{Cl}$ for the percentage point difference was greater than -10 .

With respect to pertussis antigens the per-protocol immunogenicity analysis also showed that anti-PT, anti-FHA, anti-PRN, and anti-FIM GMTs were noninferior in Group A compared with Group B (Table 8).

Few subjects $(\sim 0.2 \%)$ discontinued because of an $\mathrm{AE}$, and no deaths were reported (Table 9). Throughout the study period, 5 subjects $(0.8 \%)$ in Group A and 5 subjects $(0.8 \%)$ in Group B reported nonfatal serious AEs; none were vaccine related. Regarding injection-site AEs, a higher proportion of subjects in Group A reported swelling $(14.4 \%)$ at the 9vHPV vaccination site compared with Group B (9.4\%), and the difference between the groups was statistically significant $(P=.007$; Table 10). Comparable proportions of subjects reported swelling at MCV4 and Tdap vaccination site in Group A and Group B $(P=.526$; Table 10$)$.
Most of the swelling was mild to moderate in intensity (ie, with a maximum size $<5 \mathrm{~cm}$ ) both at the $9 \mathrm{vHPV}$ vaccine injection site and the MCV4 and Tdap injection site. Most subjects in each group (Group A: 92.3\%; Group B: 91.9\%) reported a maximum temperature $<37.8^{\circ} \mathrm{C}$ $\left(<100^{\circ} \mathrm{F}\right)$ within 5 days of the first vaccination. The proportions with elevated temperatures $\left(\geq 37.8^{\circ} \mathrm{C}\right)$ within 5 days of the first vaccination were similar between the 2 groups (risk difference: $-0.4 ; 95 \%$ confidence interval: -3.5 to 2.7 ). No subject became pregnant during the study.

\section{DISCUSSION}

This study demonstrated that when the first dose of $9 \mathrm{vHPV}$ vaccine is administered concomitantly with MCV4 and Tdap at a separate injection site, the immune response to all vaccine components is noninferior to the immune response achieved when the 3 vaccines are administered nonconcomitantly. Specifically, GMTs to all 9 vaccine HPV types were noninferior in Group A compared with Group B, and all subjects in both vaccination groups seroconverted after the third dose of $9 \mathrm{vHPV}$ vaccine. Also, antibody responses to $N$ meningitidis serogroups $\mathrm{A} / \mathrm{C} / \mathrm{Y} / \mathrm{W}-135$, diphtheria, tetanus, and pertussis at 4 weeks after administration of MCV4 and Tdap were noninferior in Group A compared with Group B.

Concomitant administration of the first dose of 9vHPV vaccine with MCV4 and Tdap was generally well tolerated. The proportion of subjects reporting injection site AEs postvaccination 1 was similar in the concomitant vaccination group (85.3\%) and the nonconcomitant vaccination group (85.1\%). Significantly more subjects reported swelling at the

TABLE 7 Estimated Percentage Point Difference in the Per-Protocol Population for Tdap for Percent of Subjects With Diphtheria and Tetanus Titers $\geq 0.1 \mathrm{IU} / \mathrm{mL}$ at 4 Weeks Postvaccination

\begin{tabular}{|c|c|c|c|c|c|c|c|}
\hline & \multicolumn{3}{|c|}{ Concomitant (Group A), ${ }^{a} N=619$} & \multicolumn{3}{|c|}{ Nonconcomitant (Group B), ${ }^{a} N=618$} & \multirow{2}{*}{$\begin{array}{l}\text { Estimated Percentage Point Difference } \\
\text { Group A - Group B }(97.5 \% \mathrm{Cl})\end{array}$} \\
\hline & $m / n$ & $\%$ & $97.5 \% \mathrm{Cl}$ & $m / n$ & $\%$ & $97.5 \% \mathrm{Cl}$ & \\
\hline \multicolumn{8}{|c|}{ Diphtheria titer $\geq 0.1 \mathrm{IU} / \mathrm{mL}$} \\
\hline Day 1 & $403 / 593$ & 68.0 & (63.5 to 72.2 ) & $391 / 564$ & 69.3 & (64.8 to 73.6 ) & \\
\hline 4 wk postvaccination & $595 / 595$ & 100 & (99.3 to 100$)$ & $566 / 566$ & 100 & (99.2 to 100$)$ & $0.0(-0.8 \text { to } 0.9)^{\mathrm{b}}$ \\
\hline \multicolumn{8}{|l|}{ Tetanus titer $\geq 0.1 \mathrm{IU} / \mathrm{mL}$} \\
\hline Day 1 & $460 / 573$ & 80.3 & (76.3 to 83.9 ) & $450 / 548$ & 82.1 & (78.1 to 85.6$)$ & \\
\hline 4 wk postvaccination & $593 / 594$ & 99.8 & (98.9 to 100$)$ & $562 / 562$ & 100 & (99.2 to 100$)$ & $-0.2(-1.2 \text { to } 0.7)^{\mathrm{b}}$ \\
\hline
\end{tabular}

$\mathrm{m}=$ number of subjects with the indicated response; $\mathrm{N}$, number of subjects randomized to the respective vaccination group who received at least 1 injection; $\mathrm{n}$, number of subjects contributing to the analysis. $\mathrm{Cl}$, confidence interval.

a Group A (concomitant administration) received a 0.5-mL dose of 9vHPV vaccine at day 1, month 2, and month 6 and MCV4 and Tdap on day 1; Group B (nonconcomitant administration) received 9vHPV vaccine as Group A and Tdap-IPV vaccine at month 1

b $P$ value for non-inferiority $<0.001$ for all antigens. The non-inferiority criterion for endpoints reported in this table was defined as statistically less than 10 percentage points decrease in Group A compared with Group B. Non-inferiority of percent with titer $\geq 0.1 \mathrm{IU} / \mathrm{mL}$ in Group A relative to Group B was demonstrated if the lower limit of the $97.5 \%$ Cl for the percentage point difference was greater than -10 
TABLE 8 Antipertussis GMTs and Estimated Fold Difference at 4 Weeks Postvaccination in the Per-Protocol Population for Tdap

\begin{tabular}{|c|c|c|c|c|c|c|c|}
\hline & \multicolumn{3}{|c|}{ Concomitant (Group A), ${ }^{a} N=619$} & \multicolumn{3}{|c|}{ Nonconcomitant (Group B), ${ }^{a} N=618$} & \multirow{2}{*}{$\begin{array}{l}\text { Estimated Fold Difference } \\
\text { Group A/Group B }(97.5 \% \mathrm{Cl})\end{array}$} \\
\hline & $n$ & Estimated GMT (ELU/m) & $97.5 \% \mathrm{Cl}$ & $n$ & Estimated GMT (ELU/m) & $97.5 \% \mathrm{Cl}$ & \\
\hline \multicolumn{8}{|l|}{ Anti-PT } \\
\hline Day 1 & 594 & 7.5 & (6.8 to 8.2 ) & 564 & 7.1 & (6.5 to 7.8 ) & \\
\hline 4 wk postvaccination & 595 & 28.5 & (25.8 to 31.5 ) & 566 & 35.7 & (32.3 to 39.6 ) & $0.80(0.69 \text { to } 0.92)^{\mathrm{b}}$ \\
\hline \multicolumn{8}{|l|}{ Anti-FHA } \\
\hline Day 1 & 594 & 33.0 & (30.4 to 35.8 ) & 564 & 32.8 & (30.1 to 35.7 ) & \\
\hline 4 wk postvaccination & 595 & 184.1 & (171.8 to 197.4$)$ & 566 & 201.4 & (187.6 to 216.3 ) & $0.91(0.83 \text { to } 1.01)^{\mathrm{b}}$ \\
\hline \multicolumn{8}{|l|}{ Anti-PRN } \\
\hline Day 1 & 594 & 20.9 & (19.2 to 22.8 ) & 564 & 20.4 & (18.6 to 22.4 ) & \\
\hline 4 wk postvaccination & 595 & 328.4 & (300.6 to 358.8 ) & 566 & 344.0 & (314.2 to 376.7 ) & $0.95(0.84 \text { to } 1.08)^{\mathrm{b}}$ \\
\hline \multicolumn{8}{|l|}{ Anti-FIM } \\
\hline Day 1 & 594 & 16.1 & (14.3 to 18.2 ) & 564 & 15.8 & (13.9 to 18.1$)$ & \\
\hline 4 wk postvaccination & 595 & 653.0 & (556.0 to 767.1$)$ & 566 & 681.4 & (577.8 to 803.7 ) & $0.96(0.76 \text { to } 1.21)^{\mathrm{b}}$ \\
\hline
\end{tabular}

9vHPV vaccine injection site after the first vaccination in the concomitant group. Injection-site swelling at the 9vHPV vaccine injection site was mostly mild to moderate in intensity. Moreover, there were few discontinuations due to an AE. Thus, the finding of increased rates of injection-site swelling is likely to be of minor clinical significance. In another study that investigated concomitant administration of 9vHPV vaccine with a diphtheria/tetanus/ pertussis/polio vaccine, more subjects also reported swelling at the 9vHPV vaccine injection site in the concomitant vaccination group. ${ }^{19}$ This suggests that the increase in injection-site swelling may be due to the concomitant administration of diphtheria/tetanus/ pertussis antigens (rather than meningococcal antigens) with 9vHPV vaccine.

The results of this study are similar to those of a previous study of concomitant administration of the quadrivalent HPV (types 6/11/16/ 18) vaccine and MCV4 and Tdap,

TABLE 9 AEs Reported Day 1 Through 15 After the Respective Vaccination Visit

\begin{tabular}{|c|c|c|c|c|c|c|}
\hline & \multicolumn{2}{|c|}{ Postvaccination $1^{a}$} & \multicolumn{2}{|c|}{ Postvaccination $2^{\mathrm{b}}$} & \multicolumn{2}{|c|}{ Postvaccination $3^{b}$} \\
\hline & $\begin{array}{l}\text { Concomitant } \\
(\text { Group } A)^{C}\end{array}$ & $\begin{array}{l}\text { Nonconcomitant } \\
\text { (Group B) }^{\mathrm{c}}\end{array}$ & $\begin{array}{l}\text { Concomitant } \\
(\text { Group } A)^{\mathrm{c}}\end{array}$ & $\begin{array}{l}\text { Nonconcomitant } \\
\left(\text { Group B) }^{\mathrm{c}}\right.\end{array}$ & $\begin{array}{l}\text { Concomitant } \\
(\text { Group A })^{\mathrm{C}}\end{array}$ & $\begin{array}{l}\text { Nonconcomitant } \\
\text { (Group B) }^{\mathrm{c}}\end{array}$ \\
\hline Subjects with follow-up, $n$ & 613 & 611 & 591 & 593 & 583 & 581 \\
\hline Subjects with $\geq 1 \mathrm{AE}, n$ (\%) & $523(85.3)$ & $520(85.1)$ & 307 (51.9) & $299(50.4)$ & $323(55.4)$ & $307(52.8)$ \\
\hline Injection-site & $496(80.9)$ & $491(80.4)$ & $276(46.7)$ & $276(46.5)$ & $304(52.1)$ & $281(48.4)$ \\
\hline Systemic & $264(43.1)$ & $259(42.4)$ & $95(16.1)$ & $89(15.0)$ & $86(14.8)$ & $94(16.2)$ \\
\hline Serious $^{d}$ & $1^{\mathrm{e}}(0.2)$ & $1^{f}(0.2)$ & $1^{g}(0.2)$ & $0(0.0)$ & $1^{\mathrm{h}}(0.2)$ & $0(0.0)$ \\
\hline Serious vaccine-related & $0(0.0)$ & $0(0.0)$ & $0(0.0)$ & $0(0.0)$ & $0(0.0)$ & $0(0.0)$ \\
\hline Discontinued due to an $\mathrm{AE}, n(\%)$ & $0(0.0)$ & $1(0.2)$ & $1(0.2)$ & $0(0.0)$ & $0(0.0)$ & $0(0.0)$ \\
\hline Discontinued due to death, $n(\%)$ & $0(0.0)$ & $0(0.0)$ & $0(0.0)$ & $0(0.0)$ & $0(0.0)$ & $0(0.0)$ \\
\hline
\end{tabular}

All subjects who received $\geq 1$ injection and had follow-up data were included in the primary analysis of safety. The number and percent of subjects reporting the following were compared between Group A and Group B: systemic AEs on days 1 through 15 after any vaccination and the number of subjects reporting clinical serious AEs from days 1 to 15 after any vaccination or vaccine-related clinical serious AE at any time during the study.

a Postvaccination 1 is defined as the follow-up period for safety after vaccination at day 1 and after vaccination/visit at month 1 . These data represent AEs reported for the 2 vaccines combined

b Because 9vHPV vaccine was the only vaccine administered in a 3-dose regimen, AEs reported postvaccination 2 and 3 would be specific to 9vHPV vaccination.

${ }^{c}$ Group A (concomitant administration) received a $0.5-\mathrm{mL}$ dose of 9vHPV vaccine at day 1, month 2, and month 6 and a 0.5 -mL dose of MCV4 and Tdap on day 1; Group B (nonconcomitant administration) received 9vHPV vaccine as Group A and MCV4 and Tdap at month 1. Two subjects randomized into the nonconcomitant group received 9vHPV vaccine at day 1 but did not receive MCV4 and Tdap at month 1 and are excluded from this table.

d There were 7 additional serious AE reported outside the day 1 to 15 time window. Group A: appendicitis 27 d postdose 2 of 9vHPV vaccine (5-d duration); appendicitis 93 d postdose 2 of 9vHPV vaccine (4-d duration). Group B: appendicitis $61 \mathrm{~d}$ postdose 1 of 9vHPV vaccine (1.3-wk duration); orthostatic hypotension $193 \mathrm{~d}$ postdose 1 of 9vHPV vaccine (3-d duration); depression 40-d postdose 2 of 9vHPV vaccine (3-d duration); dengue fever $47 \mathrm{~d}$ postdose 2 of 9vHPV vaccine (1.6-wk duration); bronchitis $127 \mathrm{~d}$ postdose 2 of $9 \mathrm{vHPV}$ vaccine (6-d duration). Thus, throughout the study period, there were 11 serious non-vaccine-related AEs reported for 10 subjects: 5 subjects (0.8\%) in Group A and 5 subjects (0.8\%) in Group B (1 subject experienced 2 serious AEs: gastroenteritis and orthostatic hypotension). All serious AEs were considered not related to any of the 3 study vaccines by the study investigators (investigators were instructed to assign causality to AEs on the basis of exposure, time course, likely cause, and consistency with the vaccine's known profile; vaccine-related AEs were those that were determined by the investigator to be possibly, probably, or definitely vaccine related).

e Seroma 9 d postdose 1 of 9vHPV vaccine (4.3-wk duration).

${ }^{f}$ Gastroenteritis $6 \mathrm{~d}$ postdose 1 of 9vHPV vaccine (2-d duration).

$\mathrm{g}$ Affective disorder $1 \mathrm{~d}$ postdose 2 of 9vHPV vaccine (1.1-wk duration)

h Testicular torsion 8 d postdose 3 (2-d duration). 
TABLE 10 Injection-Site AEs Prompted for on the VRC

\begin{tabular}{lcccc}
\hline & $\begin{array}{c}\text { Concomitant } \\
\text { (Group A) }^{\text {a }}\end{array}$ & $\begin{array}{c}\text { Nonconcomitant } \\
\text { (Group B) }^{\text {a }}\end{array}$ & $\begin{array}{c}\text { \% Difference Group A } \\
\text { vs Group B (95\% Cl) }\end{array}$ & $P$ \\
\hline 9vHPV injection site (reported d 1-5 postdose 1) & & & & \\
Subjects contributing to the analysis, $n$ & 611 & 609 & & \\
$\quad$ Erythema, $n$ (\%) & $61(10.0)$ & $54(8.9)$ & $1.1(-2.2$ to 4.4$)$ & .505 \\
Pain, $n$ (\%) & $356(58.3)$ & $335(55.0)$ & $3.3(-2.3$ to 8.8) & .251 \\
$\quad$ Swelling, $n$ (\%) & $88(14.4)$ & $57(9.4)$ & $5.0(1.4$ to 8.7) & .007 \\
MCV4 and Tdap injection site (reported d 1-5 postvaccination) & & & \\
$\quad$ Subjects contributing to the analysis, $n$ & 611 & 598 & & \\
$\quad$ Erythema, $n$ (\%) & $159(26.0)$ & $152(25.4)$ & $0.6(-4.3$ to 5.5) & .810 \\
$\quad$ Pain, $n$ (\%) & $439(71.8)$ & $406(67.9)$ & $4.0(-1.2$ to 9.1) & .134 \\
Swelling, $n$ (\%) & $188(30.8)$ & $174(29.1)$ & $1.7(-3.5$ to 6.8) & .526 \\
\hline
\end{tabular}

All subjects who received $\geq 1$ injection and had follow-up data were included in the primary analysis of safety. The number and percent of subjects reporting injection-site AEs were compared between Group A and Group B. Risk differences, $95 \% \mathrm{Cls}$ and $P$ values were calculated for injection site AEs between day 1 and day 5 for both groups. $\mathrm{Cl}$, confidence interval.

a Group A (concomitant administration) received a $0.5-\mathrm{mL}$ dose of 9vHPV vaccine at day 1, month 2, and month 6 and a 0.5-mL dose of MCV4 and Tdap on day 1; Group B (nonconcomitant administration) received 9vHPV vaccine as Group A and MCV4 and Tdap at month 1. Two subjects randomized into the nonconcomitant group received 9vHPV vaccine at day 1 but did not did not receive MCV4 and Tdap at month 1 and are excluded from this table.

which showed that concomitant administration of dose 1 of quadrivalent HPV vaccine and MCV4 and Tdap was generally well tolerated and did not interfere with the antibody response to any of the vaccine antigens; similar to this study, increased rates in injection-site swelling were also noted in the concomitant vaccination group. ${ }^{20}$

The primary limitation of this study was its unblinded nature. As such, safety assessment could have been biased toward an overestimation of AEs being reported in the concomitant vaccination group because subjects who are receiving 2 injections on the same day may more likely report injection-site or systemic AEs compared with subjects who receive only 1 injection. Another limitation of this study is that the coadministration of Tdap and MCV4 was assessed only with the first dose of HPV vaccine and not with subsequent doses.

Even though only 1 Tdap vaccine was assessed in this study, one can reasonably assume that the results may be generalizable to other Tdap vaccines. Differences in antigen and aluminum dose between diphtheria/tetanus/ pertussis vaccines such as Adacel, Boostrix-IPV, or Repevax are relatively modest and therefore expected to have limited impact on 9vHPV vaccine immunogenicity. A limited impact on 9vHPV vaccine immunogenicity is unlikely to have clinical significance: the $9 \mathrm{vHPV}$ vaccine efficacy findings in young women were extended to adolescents based on the demonstration of noninferior anti-HPV responses ${ }^{7}$; anti-HPV responses in adolescents were actually much higher than in young women. Even if concomitant administration of a different diphtheria/tetanus/pertussis vaccine were associated with a small decrease in 9vHPV vaccine immunogenicity, anti-HPV responses would still be substantially higher than in young women, and therefore protection elicited by $9 \mathrm{vHPV}$ vaccine would not be compromised.

When the study was initiated in 2009 , other meningococcal serotype A/C/ $\mathrm{Y} / \mathrm{W}-135$ vaccines (Menveo and Nimenrix) had not yet been licensed. Although Menactra, Menveo, and Nimenrix contain similar amounts of polysaccharide and carrier protein, they use different carrier proteins; thus, it is difficult to speculate whether results obtained in this study can be extrapolated to other meningococcal vaccines. Regulatory guidelines caution against such extrapolation for conjugated polysaccharide vaccines. ${ }^{21}$
This study demonstrates that concomitant administration of $9 \mathrm{vHPV}$ vaccine and MCV4 and Tdap was generally well tolerated and the immune responses to components of either vaccine were noninferior compared with nonconcomitant administration. Providing vaccinations to adolescents is challenging because they make infrequent health care visits. Concomitant administration would minimize the number of visits required to deliver each vaccine individually and therefore facilitate adherence to recommended vaccination regimens. In the United States, coverage for the first dose of HPV vaccine remains substantially lower (by $\sim 20-25$ percentage points) than coverage for other vaccines recommended by the Advisory Committee on Immunization Practices for children 11 to 12 years of age..$^{22,23}$ It is estimated that coadministration of HPV vaccine with other vaccines such as diphtheria, tetanus, pertussis, meningococcal conjugate, and influenza vaccines could increase coverage for the first dose of HPV vaccine to $>90 \% .^{24}$

\section{ACKNOWLEDGMENTS}

The authors thank Frank Dutko, PhD, Ms Danielle Mancaruso, and Ms Karyn Davis (Merck \& Co, Inc, Kenilworth, NJ) for assistance in the preparation of the manuscript.

\section{ABBREVIATIONS}

9vHPV: 9-valent HPV (6/11/16/ 18/31/33/45/52/58) L1 virus-like particle vaccine

AEs: adverse events

ELISA: enzyme-linked immunosorbent assay

FHA: filamentous hemagglutinin

FIM: fimbriae $2 / 3$

GMT: geometric mean titers

HPV: human papillomavirus

PRN: pertactin

PT: pertussis toxin

VRC: vaccination report card 
Drs Huicho and Luxembourg substantially contributed to interpretation of the results and drafting of the manuscript and reviewed and revised the manuscript for important intellectual content; Dr Sobanjo-ter Meulen substantially contributed to conception, design and planning of the study and interpretation of the results and reviewed and revised the manuscript for important intellectual content; and all authors approved the final manuscript as submitted.

This trial has been registered at www.clinicaltrials.gov (identifier NCT00988884).

www.pediatrics.org/cgi/doi/10.1542/peds.2014-4199

DOI: $10.1542 /$ peds.2014-4199

Accepted for publication Jun 11, 2015

Address correspondence to Andrea Schilling, MD, Facultad de Medicina Clinica Alemana-Universidad del Desarrollo, Santiago, Chile. E-mail: dra.andrea.schilling@ gmail.com

PEDIATRICS (ISSN Numbers: Print, 0031-4005; Online, 1098-4275)

Copyright (C) 2015 by the American Academy of Pediatrics

FINANCIAL DISCLOSURE: Dr Schilling has received research support for this clinical study and other HPV vaccine-related studies from Merck and honoraria outside the submitted work from Merck-Chile, Grunenthal-Chile, and Grunenthal-L.A. Dr Parra has received research support for this clinical study and grant support from Merck. Dr Gutierrez has received research support for this clinical study from Merck. Dr Restrepo has received research support for this clinical study from Merck and has received compensation from Froost Laboratories for Board Membership for GARDASIL. Drs Ucros, Herrera, Engel, and Huicho have received research support for this clinical study from Merck. Dr Shew has received research support for this clinical study and other HPV vaccine-related studies from Merck. Mr Maansson is a former employee of Merck \& Co., Inc. Ms Caldwell is a former employee of Merck \& Co., Inc., and holds stock and stock options in Merck \& Co., Inc. Dr Luxembourg is a current employee receiving salary from Merck \& Co., Inc., and holds stock and stock options in Merck \& Co., Inc. Ajoke Sobanjo-ter Meulen is a former employee of Merck \& Co., Inc. and now works at Novartis.

FUNDING: Supported by Merck \& Co., Inc, Kenilworth, NJ.

POTENTIAL CONFLICT OF INTEREST: Dr Schilling has received research support for this clinical study and other HPV vaccine-related studies from Merck and honoraria outside the submitted work from Merck-Chile, Grunenthal-Chile, and Grunenthal-L.A. Dr Parra has received research support for this clinical study and grant support from Merck. Dr Gutierrez has received research support for this clinical study from Merck. Dr Restrepo has received research support for this clinical study from Merck and has received compensation from Froost Laboratories for Board Membership for GARDASIL. Drs Ucros, Herrera, Engel, and Huicho have received research support for this clinical study from Merck. Dr Shew has received research support for this clinical study and other HPV vaccine-related studies from Merck. Mr Maansson is a former employee of Merck \& Co., Inc. Ms Caldwell is a former employee of Merck \& Co., Inc., and holds stock and stock options in Merck \& Co., Inc. Dr Luxembourg is a current employee receiving salary from Merck \& Co., Inc., and holds stock and stock options in Merck \& Co., Inc. Ajoke Sobanjo-ter Meulen is a former employee of Merck \& Co., Inc. and now works at Novartis.

\section{REFERENCES}

1. Serrano B, Alemany L, Tous S, et al. Potential impact of a nine-valent vaccine in human papillomavirus related cervical disease. Infect Agent Cancer. 2012;7(1):38

2. de Sanjosé S, Alemany L, Ordi J, et al; HPV VVAP study group. Worldwide human papillomavirus genotype attribution in over 2000 cases of intraepithelial and invasive lesions of the vulva. Eur $J$ Cancer. 2013;49(16):3450-3461

3. Alemany L, Saunier M, Alvarado-Cabrero I, et al; HPV VVAP Study Group. Human papillomavirus DNA prevalence and type distribution in anal carcinomas worldwide. Int J Cancer. 2015;136(1): 98-107

4. Alemany L, Saunier M, Tinoco L, et al; HPV VVAP study group. Large contribution of human papillomavirus in vaginal neoplastic lesions: a worldwide study in 597 samples. Eur J Cancer. 2014;50(16):2846-2854

5. Lacey CJ, Lowndes CM, Shah KV. Chapter 4: burden and management of non- cancerous HPV-related conditions: HPV6/11 disease. Vaccine. 2006;24(suppl 3): S3-, 35-41

6. Joura EA, Giuliano AR, Iversen 0-E, et al; Broad Spectrum HPV Vaccine Study. A 9-valent HPV vaccine against infection and intraepithelial neoplasia in women. N Engl J Med. 2015;372(8):711-723

7. Van Damme P, Olsson SE, Block S, et al. Immunogenicity and safety of a 9-valent HPV vaccine. Pediatrics. 2015;136(1): e28-e39

8. Prescribing Information. GARDASIL9 (Human Papillomavirus 9-valent Vaccine, Recombinant). Revised: February 2015. Available at: http://www.merck.com/ product/usa/pi_circulars/g/gardasil_9/ gardasil_9_pi.pdf. Accessed April 28, 2015

9. Petrosky E, Bocchini JA Jr, Hariri S, et al; Centers for Disease Control and Prevention (CDC). Use of 9-valent human papillomavirus (HPV) vaccine: updated HPV vaccination recommendations of the advisory committee on immunization practices. MMWR Morb Mortal Wkly Rep. 2015;64(11):300-304

10. Winer RL, Lee SK, Hughes JP, Adam DE, Kiviat NB, Koutsky LA. Genital human papillomavirus infection: incidence and risk factors in a cohort of female university students. Am J Epidemiol. 2003;157(3):218-226

11. Wellings K, Collumbien M, Slaymaker E, et al. Sexual behaviour in context: a global perspective. Lancet. 2006; 368(9548):1706-1728

12. Broder KR, Cortese MM, Iskander JK, et al; Advisory Committee on Immunization Practices (ACIP). Preventing tetanus, diphtheria, and pertussis among adolescents: use of tetanus toxoid, reduced diphtheria toxoid and acellular pertussis vaccines recommendations of the Advisory Committee on Immunization Practices (ACIP). MMWR Recomm Rep 2006;55 (RR-3): 1-34 
13. European Centre for Disease Prevention and Control (ECDC) Guidance-Scientific panel on childhood immunization schedule: diphtheria-tetanus-pertussis (DTP) vaccination. 2009. Available at http://www.ecdc.europa.eu/en/ publications/Publications/0911_GUI_ Scientific_Panel_on_Childhood_ Immunisation_DTP.pdf. Accessed October 17, 2014

14. Cohn AC, MacNeil JR, Clark TA, et al; Centers for Disease Control and Prevention (CDC). Prevention and control of meningococcal disease: recommendations of the Advisory Committee on Immunization Practices (ACIP). MMWR Recomm Rep. 2013;62(RR2): $1-28$

15. Prescribing Information. Menactra meningococcal (groups A, C, Y and W-135) polysaccharide diphtheria toxoid conjugate vaccine). Revised: August 2014 Available at: http://www.sanofipasteur. us/vaccines. Accessed October 17, 2014

16. Prescribing Information. Adacel (tetanus toxoid, reduced diphtheria toxoid and acellular pertussis vaccine adsorbed). Revised: March 2014. Available at: http:// www.sanofipasteur.us/vaccines.

Accessed 0ctober 17, 2014

17. Roberts C, Green T, Hess E, et al. Development of a human papillomavirus competitive luminex immunoassay for 9 HPV types. Hum Vaccin Immunother. 2014;10(8):2174-103 doi:10.4161/ hv. 29205

18. Miettinen 0, Nurminen M. Comparative analysis of two rates. Stat Med. 1985; 4(2):213-226

19. Kosalaraksa P, Mehlsen J, Vesikari T, et al. An open-label, randomized study of a 9-valent human papillomavirus vaccine given concomitantly with diphtheria, tetanus, pertussis and poliomyelitis vaccines to healthy adolescents 11-15 years of age. Pediatr Infect Dis J. 2015; 34(6):627-634 doi:10.1097/ INF.0000000000000694

20. Reisinger KS, Block SL, Collins-0gle M, et al; Protocol 025 Investigators. Safety, tolerability, and immunogenicity of
Gardasil given concomitantly with Menactra and Adacel. Pediatrics. 2010; 125(6):1142-1151

21. Vaccine Working Party (VWP) Conclusions from the Workshop on $\mathrm{Co}_{-}$ administration of Vaccines held on 31 Jan-1 Feb 2006. January 16, 2007. Doc. Ref: EMEA/CHMP/VWP/14684/2007. Available at: http://www.ema.europa.eu/ docs/en_GB/document_library/0ther/ 2009/11/WC500015459.pdf. Accessed June 18, 2015

22. Schuchat A. HPV "coverage." N Engl J Med. 2015;372(8):775-776

23. Centers for Disease Control and Prevention. Immunization schedules: birth-18 years and catch-up immunization schedules. Available at: http://www.cdc.gov/vaccines/schedules/ hcp/child-adolescent.html. Accessed March 22, 2015

24. Noronha AS, Markowitz LE, Dunne EF. Systematic review of human papillomavirus vaccine coadministration. Vaccine. 2014;32(23):2670-2674 


\section{Coadministration of a 9-Valent Human Papillomavirus Vaccine With Meningococcal and Tdap Vaccines}

Andrea Schilling, Mercedes Macias Parra, Maricruz Gutierrez, Jaime Restrepo, Santiago Ucros, Teobaldo Herrera, Eli Engel, Luis Huicho, Marcia Shew, Roger Maansson, Nicole Caldwell, Alain Luxembourg and Ajoke Sobanjo ter Meulen Pediatrics; originally published online August 3, 2015;

DOI: $10.1542 /$ peds. 2014-4199

\section{Updated Information \& Services}

\section{Supplementary Material}

Subspecialty Collections

Permissions \& Licensing

Reprints including high resolution figures, can be found at: http://pediatrics.aappublications.org/content/early/2015/07/28 /peds.2014-4199

Supplementary material can be found at: http://pediatrics.aappublications.org/content/suppl/2015/07/2 8/peds.2014-4199.DCSupplemental.html

This article, along with others on similar topics, appears in the following collection(s):

Infectious Diseases

http://pediatrics.aappublications.org/cgi/collection/infectious diseases_sub

\section{$\overline{\text { Vaccine/Immunization }}$}

http://pediatrics.aappublications.org/cgi/collection/vaccine:im munization_sub

\section{Sexually Transmitted Infections}

http://pediatrics.aappublications.org/cgi/collection/sexually_t ransmitted_infections_new_sub

Information about reproducing this article in parts (figures, tables) or in its entirety can be found online at: http://pediatrics.aappublications.org/site/misc/Permissions.xh tml

Information about ordering reprints can be found online: http://pediatrics.aappublications.org/site/misc/reprints.xhtml

PEDIATRICS is the official journal of the American Academy of Pediatrics. A monthly publication, it has been published continuously since 1948. PEDIATRICS is owned, published, and trademarked by the American Academy of Pediatrics, 141 Northwest Point Boulevard, Elk Grove Village, Illinois, 60007. Copyright @ 2015 by the American Academy of Pediatrics. All rights reserved. Print ISSN: 0031-4005. Online ISSN: 1098-4275.

\section{American Academy of Pediatrics}

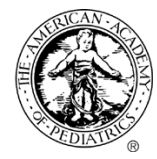




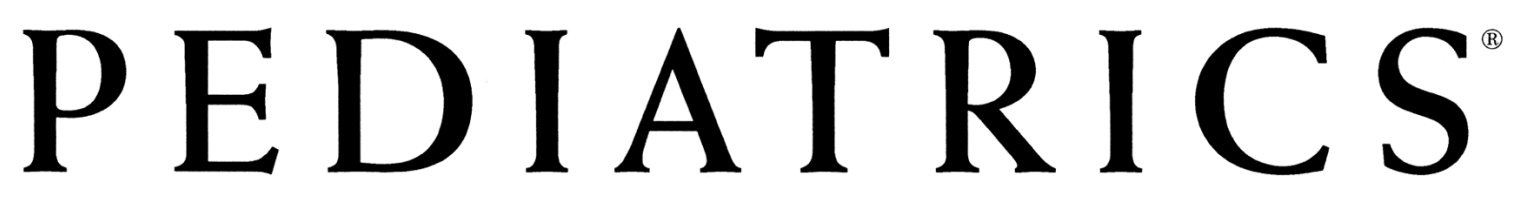

OFFICIAL JOURNAL OF THE AMERICAN ACADEMY OF PEDIATRICS

\section{Coadministration of a 9-Valent Human Papillomavirus Vaccine With Meningococcal and Tdap Vaccines}

Andrea Schilling, Mercedes Macias Parra, Maricruz Gutierrez, Jaime Restrepo, Santiago Ucros, Teobaldo Herrera, Eli Engel, Luis Huicho, Marcia Shew, Roger Maansson, Nicole Caldwell, Alain Luxembourg and Ajoke Sobanjo ter Meulen Pediatrics; originally published online August 3, 2015;

DOI: $10.1542 /$ peds.2014-4199

The online version of this article, along with updated information and services, is located on the World Wide Web at:

http://pediatrics.aappublications.org/content/early/2015/07/28/peds.2014-4199

PEDIATRICS is the official journal of the American Academy of Pediatrics. A monthly publication, it has been published continuously since 1948. PEDIATRICS is owned, published, and trademarked by the American Academy of Pediatrics, 141 Northwest Point Boulevard, Elk Grove Village, Illinois, 60007. Copyright (@) 2015 by the American Academy of Pediatrics. All rights reserved. Print ISSN: 0031-4005. Online ISSN: 1098-4275. 